\title{
Parasitic Aware Routing Methodology based on Higher Order RLCK Moment Metrics
}

\author{
Amitava Bhaduri and Ranga Vemuri \\ Department of ECECS \\ University of Cincinnati, Cincinnati, $\mathrm{OH} 45221$
}

$\{$ bhadhua, ranga\}@ececs.uc.edu

\begin{abstract}
In the multi-GHz frequency domain, inductive and capacitive parasitics of interconnects can cause significant 'ringing' or overdamping, which may lead to false switching or increased delay. Routing techniques which rely on wire length reduction or coupling capacitance minimization are unable to obtain the best routing solution. In this paper, the inductive and capacitive interactions of the wires are introduced through a 'moment' based cost function. This higher order RLCK moment metric guides the routing process of long wires, ensuring that the chosen solution has the best trade-off between ringing and delay under a monotone signal response. Another significant departure from existing routing methodologies is to account for the signal direction in the nets which may increase or decrease the effective inductive and capacitive parasitics.
\end{abstract}

\section{INTRODUCTION AND MOTIVATION}

Inductive and capacitive parasitics, both self and mutual, which were ignored in the realm of low-frequency designs, become important at high frequencies and are a dominant factor in determining interconnect delay and crosstalk of the circuit. Traditional 'maze' routing always finds the shortest path, which may lead to significant parallel runs between two neighboring wires giving rise to considerable amount of inductive and capacitive coupling. Recent crosstalk-aware routing methods [1] [2] have used coupling capacitance as a crosstalk measure. But, self and mutual inductive effects like ringing, can cause signal oscillations (overshoots and undershoots), leading to a greater settling time delay. We provide a motivating example in this regard.

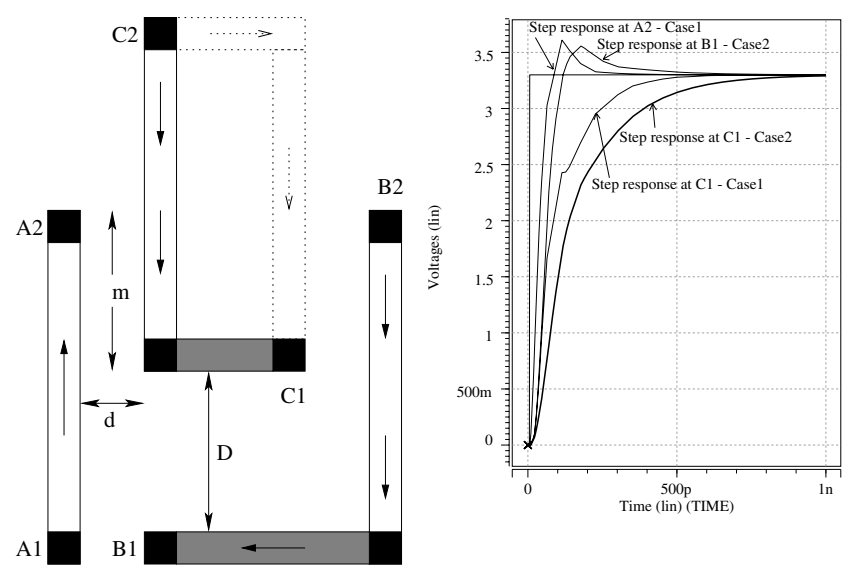

Figure 1: (A) Possible 1-bend routes (B) Signal Response at C1
In this simple example (Figure 1(A)), we consider only 1-bend routes ('L' shaped) for simplicity. We consider a situation when nets $\mathrm{A} 1-\mathrm{A} 2$ and $\mathrm{B} 1-\mathrm{B} 2$ have already been routed. We now try to determine the route for net $\mathrm{C} 1-\mathrm{C} 2$. There are two possible candidate routes for this net.

Case1: The lower-L route separated by distance 'd' that couples with A1-A2 for a length of ' $m$ ' and

Case2: The (dotted) upper-L route separated by distance 'd' that couples with B1-B2 for a length of ' $m$ '.

(Note: the distance ' $\mathrm{D}$ ' is greater than the threshold distance below which coupling is considered, hence the lower-L route does not couple with B1-B2).

As the coupling capacitance $\left(C c \propto \frac{m}{d}\right)$ is equal in both the cases, it becomes difficult to pick the best route from these two solutions. In our 'moment' driven routing cost function, we account for factors such as self and mutual inductance $(\mathrm{L}, \mathrm{K})$, direction of signal propagation, in addition to resistance $(\mathrm{R})$ and self $(\mathrm{C})$ and coupling capacitance $(\mathrm{Cc})$ between the neighboring wires, to find the best candidate route. In Case 1 , we see that the current flows in opposite direction (odd-mode of propagation) and in Case2, the current flows in the same direction (even-mode of propagation). From the signal response at $\mathrm{C} 1$ in Figure 1(B), we observe that Case2 leads to a more overdamped response compared to Case1, leading to a greater net delay. Thus, Case1 (lower-L) is the better routing choice for net $\mathrm{C} 1 \mathrm{C} 2$ as it's response is closer to the 'critically-damped' condition compared to Case2, leading to faster response.

A higher order moment-based routing methodology is proposed, governed by a cost function, which accounts for the R,L,C,K parasitics between neighboring wires and converges to a routing solution, after a suitable ringing-delay trade off. A line-search routing methodology has been used and customized to incorporate our cost function. Since our focus is to route long wires connecting different chips distributed over the entire silicon substrate, we do not use maze routing, due to its expensive memory requirements.

\section{RELATED WORK}

The authors in [3] have used a simple congestion estimation technique in their global routing methodology. They have not allowed the number of route edges across a global bin edge exceed the edge capacity. Similar congestion estimation strategy have been used by the authors in [4], [5] with competing cost function formulation where the routing cost increased either linearly or abruptly with congestion. In [1], a routing technique has been proposed that is coupling aware only in terms of capacitance. Another work in the area of crosstalk reduction has been done in [2], [6] where the parasitic coupling capacitance occurs in the cost function formulation of the routing methodology and during layer track assignment respectively. The authors in [7], [8], [9] have developed various perfor- 
mance driven routing strategies using a simple Elmore (RC) delay model for the interconnects. Cong et al. [10], have used higher order RLC model in their MINOTAUR global router but they have not considered interwire inductive and capacitive coupling. Though all of the above routing methodologies have their own merits, none of them accounts for all the R,L,C,K parasitics during routing.

\section{RINGING AND WAVEFORM RESPONSE}

At high frequencies, the inductive effect of the interconnects gives rise to a transmission-line phenomenon called 'ringing' that can inadvertently cause the circuit to transit to a wrong state and cause logic failure. This phenomenon is shown in Figure 2(C) and is well explained in [11]. Figure 2(C) shows the signal response at the output of the 'aggressor' line of the circuit models in Figure 2(A) and 2(B). Ringing is either pronounced or subdued depending on the even or odd mode of current propagation in the aggressor and victim wires. The repeated 'overshoots' and 'undershoots' adversely affect the delay of the line as it increases the 'settling-time' of the signal response. In addition to the rise time $\left(t_{r}\right)$ of the signal, the settling time $\left(t_{s}\right)$ has to be considered to obtain the actual wire delay $\left(t_{d}\right)$ [11], [10], resulting in the expression, $t_{d}=t_{r}+t_{s}$. The
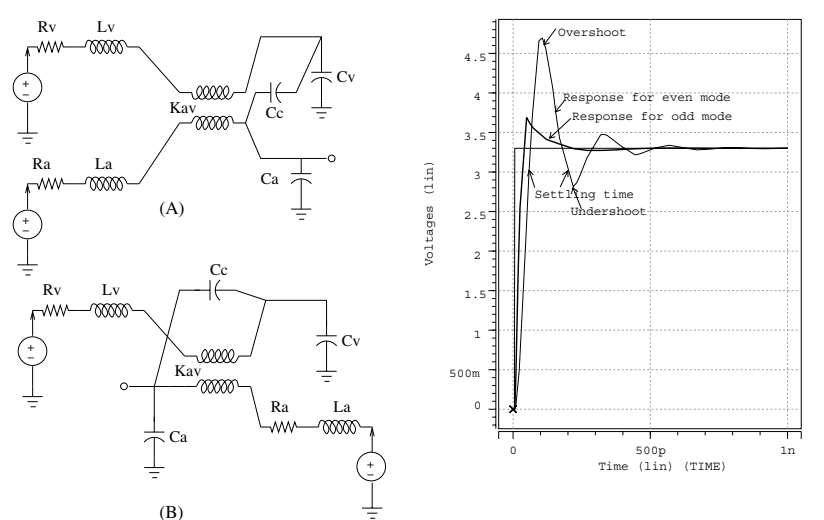

Figure 2: (A) Even-mode (B) odd-mode interaction (C) Ringing phenomenon in output waveform response

RC Elmore delay model used in previous routing papers, is insufficient to account for the high frequency inductive parasitics of the interconnects and can lead to inaccuracy. In order to account for the self and mutual inductive effects we resort to higher order moments of the impulse response to create an accurate delay approximation. Using the Laplace transform of the impulse response and with the help of the concept of central moments from probability theory as described in [12], the second and third order moments, measures of dispersion and skewness, are derived as in Eqn. 1 and Eqn. 2.

$$
\begin{aligned}
\mu_{2} & =2 m_{2}-\frac{m_{1}^{2}}{m_{0}} \\
\text { and, } \mu_{3} & =-6 m_{3}+6 \frac{m_{1} m_{2}}{m_{0}}-2 \frac{m_{1}^{3}}{m_{0}^{2}}
\end{aligned}
$$

There will always be an inherent amount of dispersion $\left(\mu_{2}\right)$ due to line loss. In contrast, $\mu_{3}$ can be made to approach the ideal value of zero [13], which ensures minimum ringing and also less settling time $\left(t_{s}\right)$ in the expression $t_{d}=t_{r}+t_{s}$. As we also minimize $\mu_{2}$, we are able to control dispersion of the lossy interconnects, and have a better signal transition. In our cost function we minimized both $\mu_{2}$ and $\mu_{3}$ as our primary objective to obtain the best trade-off between ringing and rise time delay. More on this aspect will be discussed in Section 4.5.

\section{PROPOSED METHODOLOGY}

We propose a routing methodology, which uses the concepts introduced in Section 3, to find the best possible overall routing solution that has the least parasitic interactions and the best ringing and delay trade-off for all the nets. We describe each of the six steps in our approach and their objectives below.

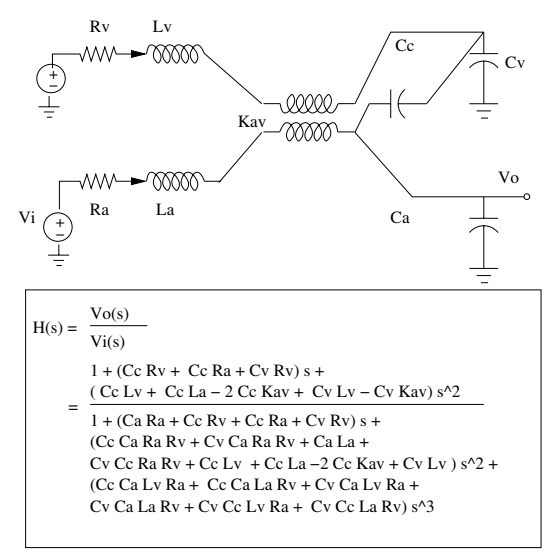

Figure 3: Transfer function of 2 interacting wires in even mode

\subsection{Transfer Function Generation}

We demonstrate a simple example in Figure 3 to generate the moment-metrics $\left(\mu_{2}\right.$ and $\left.\mu_{3}\right)$ of two interacting wires, where the top wire is the victim and the bottom wire is the aggressor. The transfer function $H(s)$ of this circuit has been generated using SAPWIN v3.0 and is shown in Figure 3. SAPWIN is essentially a symbolic transfer function generator $\left(V_{0} / V_{i}\right)$ that generates the transfer function after solving the Kirchoff's node voltage and branch current equations using modified-nodal-analysis (MNA) techniques. The important point to note in the expression of $H(s)$ is the introduction of coupling terms $\mathrm{Cc}$ and $\mathrm{K}$. The symbolic representation of the second and third order moments of this circuit is given in Eqn. 3 and Eqn. 4 respectively.

$$
\begin{aligned}
\mu_{2}= & \left(C a^{2}+2 C a C c\right) R a^{2}-2 C v C c R a R v \\
& -2 C v K a v-2 C a L a \\
\mu_{3}= & \left(2 C a^{3}+6 C a^{2} C c+6 C a C c^{2}\right) R a^{3}+\left(-6 C v C c^{2} R v\right. \\
& \left.+6 C a C c^{2} R v\right) R a^{2}+(12 C a C c K a v-12 C a C c L a \\
& -6 C v C c^{2} R v^{2}-6 C v^{2} C c R v^{2}-6 C v K a v C c \\
& \left.+6 C v C c L v-6 C a^{2} L a\right) R a-6 C v^{2} K a v R v \\
& +6 C v C c L a R v-6 C v K a v C c R v
\end{aligned}
$$

In order to come to a reasonable conclusion and an intuitive explanation from such a complex value of $\mu_{2}$ and $\mu_{3}$ we assume the capacitance and resistance of the aggressor and the victim wires to be equal, i.e, $C a=C v$ and $R a=R v$. However, we have used the entire expression of $\mu_{2}$ and $\mu_{3}$ and have not imposed the limitation of $C a=C v$ and $R a=R v$ in our methodology. The corresponding reduced equations are given below. 

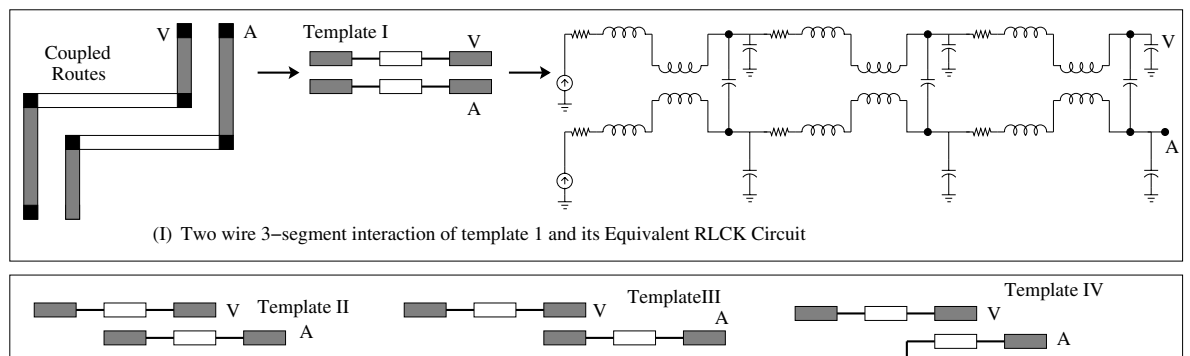

(II) Templates 2, 3 and 4 and their various interactions

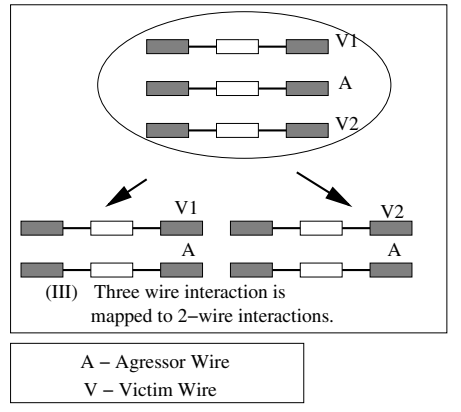

V-Victim Wire

Figure 4: Templates to determine interactions among wires and an example showing the RLCK elements for Template 1

$$
\begin{aligned}
\mu_{2}= & R a^{2} C a^{2}+2 R a^{2} C a C c-2 R a^{2} C a C c \\
& -2 C a K a v-2 C a L a \\
\Rightarrow \mu_{2}= & R a^{2} C a^{2}-2 C a(L a+K a) \\
\mu_{3}= & 2 R a^{3} C a^{3}-6 R a C a^{2} L a-6 C a^{2} K a v R a \\
\Rightarrow \mu_{3}= & 2 R a^{3} C a^{3}-6 R a C a^{2}(L a+K a v)
\end{aligned}
$$

From Eqn. 5 and Eqn. 6 we can intuitively infer that $\mu_{2}$ and $\mu_{3}$ are always positive when $L a, K a=0$, which represents lossy lines with "overdamped" response [14]. Also, $\mu_{2}$ and $\mu_{3}$ decreases monotonically as the self and mutual inductance values increases. We also notice that the effective inductance is the sum of $(L a+$ $K a v)$ signifying even mode of current propagation in the aggressor and victim wires. We can conclude that, when interconnect self and mutual inductance is not important (as is the case at sub- $\mathrm{GHz}$ frequencies) the analysis corresponds to a simple RC model with "overdamped" waveform response. Similarly, $\mu_{2} \leq 0$ and $\mu_{3} \leq 0$ signifies the inductive effects of the interconnects, which results in "ringing" as described earlier in Section 3.

Objective: The symbolic transfer function $(H(s))$ of interconnects are pre-generated using SAPWIN, which also accounts for the nearest neighbor mutual inductance and coupling capacitance terms. From this transfer function we obtain the symbolic expressions of $\mu_{2}$ and $\mu_{3}$ as shown in Section 3. The different ways an interconnect can interact with its neighboring wire will be discussed in section 4.2. The symbolic generation of the expressions of $\mu_{2}$ and $\mu_{3}$ is a one-time process and occur outside the loop of our routing algorithm. Dynamic evaluation of these symbolic expressions is made within our algorithm as the values of $\mathrm{K}$ and $\mathrm{Cc}$ changes for different candidate routes of a particular net.

\subsection{Templates for Candidate Routes}

The above analysis shows that inductive effects play a crucial role in determining the correct delay and output signal response. We have extended the above analysis to generate robust transfer functions for the candidate interconnect structures used in our router A 1-bend (L-shaped) wire is split into two segments while a 2-bend (Z-shaped) wire is split into three segments to model the nets as a simple distributed RLC network. It is important to note that during the routing process, routing of the current net also affects the waveform response of its neighboring "victim" nets.

In order to capture accurate parasitic interactions between the aggressor and the victim wires, we have generated the required templates (Figure 4) that cover the various ways the 1-bend and 2-bend interconnects can interact with each other. The $\mathrm{L}$-shaped route is a subset of Z-shaped route, hence we generate the templates with only Z-shaped nets in mind. By reducing R,L and C of any of the end segments to zero of a $\mathrm{Z}$-shaped route, we can produce the required template for a L-shaped net. The corresponding transfer functions are pre-generated for these templates and are used on the fly as deemed necessary, based on the way the candidate routes for the current net interacts with its neighboring wires. Figure 4(III) demonstrates how a 3-wire interaction for the aggressor net (A) is broken down into two, 2-wire interaction. The transfer function for each of the corresponding 2-wire interaction is then used to determine the second and third-order central moments and finally combined to generate the cumulative $\mu_{2}$ and $\mu_{3}$. For the 3 -wire case we have assumed that the victim wires at the top (V1) and the bottom (V2) do not interact with each other.

These four primitive templates or their derived reduced forms are used to capture the various possible interactions of the 1-bend and 2-bend wires with other neighboring wires.

Objective: The templates account for the various ways the Lshaped and the Z-shaped nets can couple with other L-shaped and Z-shaped wires. The templates and their symbolic transfer functions are pre-generated using SAPWIN. Using these transfer functions the central moments $\left(\mu_{2}\right.$ and $\left.\mu_{3}\right)$ for each of the templates are symbolically derived using the symbolic Maple toolbox of Matlab and are stored in a library. During the routing of a net our momentdriven routing algorithm chooses the required template from the library and evaluates its corresponding moments dynamically based on the values of $\mathrm{R}, \mathrm{L}, \mathrm{C}, \mathrm{Cc}$ and $\mathrm{K}$.

\subsection{Estimation of $R, L, C, K$}

Quick and accurate estimation of the electrical parameters R,L,C, $\mathrm{Cc}$ and $\mathrm{K}$ is made based on the wire geometry and using their corresponding analytical expressions [12]. We did not consider the coupling parasitics between orthogonal wires and have used expressions given in [15] for calculating mutual inductance of two parallel wires with unequal length. The accuracy of the self and mutual inductance $(\mathrm{L}, \mathrm{K})$ formulas with field-solver simulations is given in [15].

\subsection{Route Cost Function}

The cost function is formulated with the knowledge that routing of a particular net at any instance of the routing process may affect the signal response and delay of its neighboring wires. During the route determination process, we consider several possible candidate solutions for a net. Let us consider that there are 'n' nets that have to be routed and at a particular instance the 'i'th net needs to be routed. The following steps illustrate the cost function formulation.

1. For each of the ' $k$ ' candidate routes of net ' $i$ ', symbolic expressions of $\mu_{2}$ and $\mu_{3}$ are evaluated using estimated values of R,L,C,Cc and $\mathrm{K}$.

2. The $\mu_{2}$ 's and $\mu_{3}$ 's of all the affected nets (victims) that have already been routed are re-evaluated. 
3. For each candidate of net 'i', we compute the overall standard deviation of the already routed and the current net as given below. This gives the cumulative cost $\left(C_{\text {cost }}\right)$ for each candidate route and is a measure of the overall dispersion and skewness from the ideal value $\left(\mu_{2 i d}=0\right.$ and $\left.\mu_{3 i d}=0\right)$.

$$
C_{\text {cost }}=\sqrt{\frac{\sum_{n=1}^{i}\left(\mu_{2}^{n}-\mu_{2 i d}\right)^{2}}{i}}+\sqrt{\frac{\sum_{n=1}^{i}\left(\mu_{3}^{n}-\mu_{3 i d}\right)^{2}}{i}}
$$

4. Store the values of the cumulative cost function for each candidate route.

5. Select the candidate route having the least cumulative cost and add to the list of routed nets.
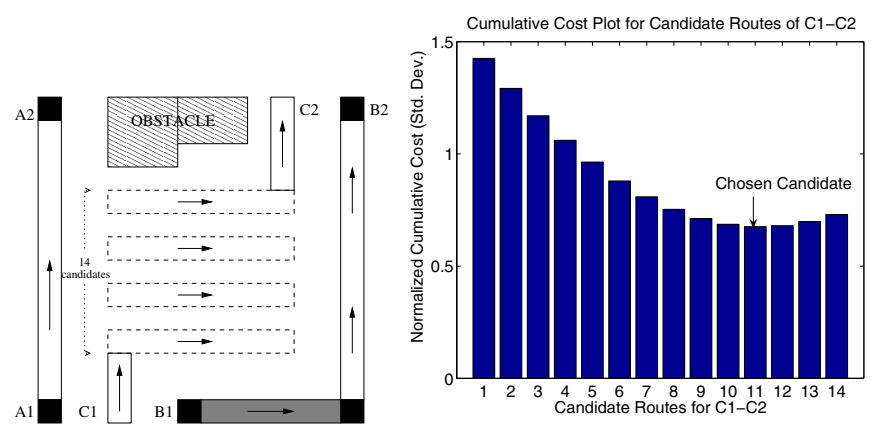

Figure 5: (A) Possible Candidate Routes of C1C2 (B) Normalized Cumulative Cost (Std. Dev) for Candidate Routes (C1C2)

The cost funtion thus gives a snapshot of the cumulative performance in terms of second and third-order central moments of all the nets up to the current net 'i'.

Figure 5(A) and 5(B) illustrates the cost function flow. Out of the 14 candidates, the cost function chooses candidate 11 as the routing solution for net $\mathrm{C} 1 \mathrm{C} 2$, which yields the least cumulative cost [16]. This implies that the chosen candidate obtains the best trade-off between ringing and delay among all other candidates and provides the least distortion to its affected neighbors A1A2 and B1B2.

Objective: The objective of developing such a cost function can be understood from Section 4.5 below.

\subsection{Ringing and Delay Trade-off}

As stated in Section 3, minimizing the second central moment $\mu_{2}$, results in reduction of the spread (dispersion) of the waveform response thereby obtaining an output response with quicker rise time $\left(t_{r}\right)$. But, the faster transition results in more ringing and the signal suffers from repeated overshoots and undershoots. Though the rise time may prove to be beneficial, the increased settling time adversely affects the signal delay $\left(t_{d}\right)$ [10], [11]. To minimize ringing and decrease the settling time delay $\left(t_{s}\right)$ we also try to minimize the third central moment $\left(\mu_{3}\right)$ sacrificing a bit on the transition rate of the signal. We formulate our cost function as given below.

$$
\begin{aligned}
S_{m o m} & =\sqrt{\frac{\sum_{n=1}^{i}\left(\mu_{2}^{n}-\mu_{2 i d}\right)^{2}}{i}} \\
T_{m o m} & =\sqrt{\frac{\sum_{n=1}^{i}\left(\mu_{3}^{n}-\mu_{3 i d}\right)^{2}}{i}} \\
C_{\text {cost }} & =S_{m o m}+\alpha T_{m o m}
\end{aligned}
$$

$S_{m o m}$ and $T_{m o m}$ in Eqn. 7 and Eqn. 8 represents the overall standard deviation of all the affected victim nets that have already been routed and the current net 'i'. Note that we have used normalized values of $S_{m o m}$ and $T_{m o m}$ to get $C_{\text {cost }}$.

Objective: The final $C_{\text {cost }}$ is evaluated by taking a weighted sum of $S_{m o m}$ and $T_{m o m}$, which ensures a relative trade-off between ringing and transition delay[13]. The $C_{\text {cost }}$ of different candidates of net ' $i$ ' are evaluated and the one that yields the minimum cost and closest to the ideal value of 0 is chosen as the best route. In our work we have given equal importance to ringing and delay minimization of a net, and have chosen $\alpha=1$ in Eqn. 9 .

\subsection{Moment-driven Routing Technique}

In our routing methodology (Figure 6) the possible candidate routes of a net are generated, similar to the level 0 and level 1 lines of the standard line search algorithm, which has been tailored to include our 'moment driven' cost function. As the routing of nets will eventually be guided by our cost function, we did not use a maze router because of its expensive memory requirements and which may not be suitable for routing multi-chip modules over a large chip area. Any ordering of nets can be handled by the router

Figure 6: Template-based moment driven routing methodology

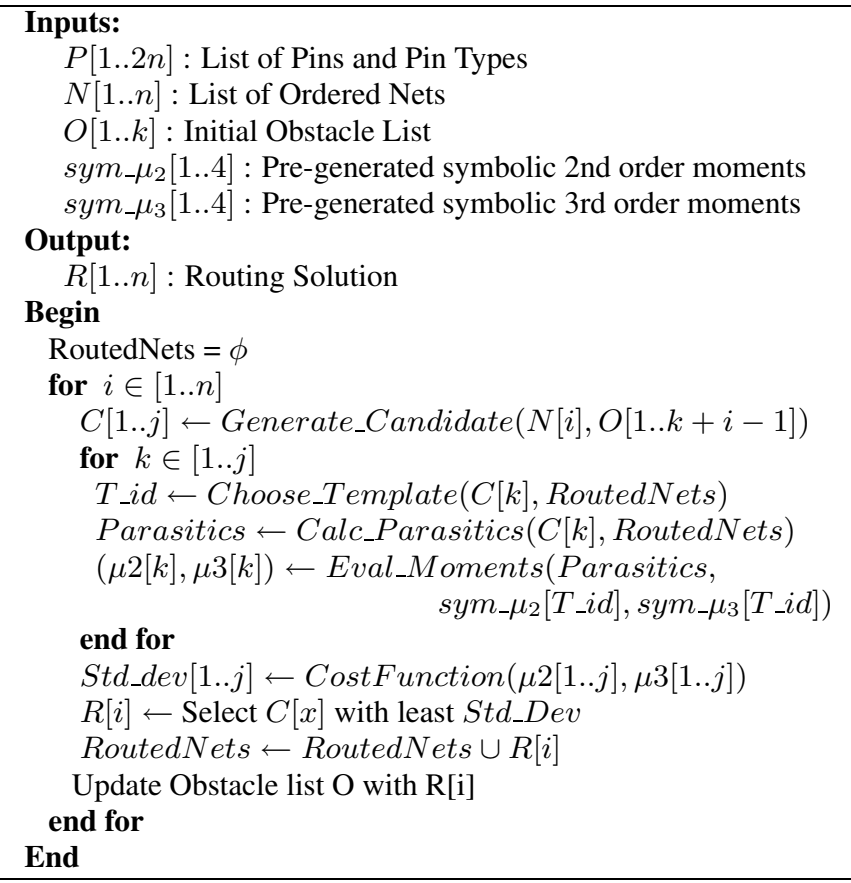

and should be given as an input to the program. The direction of signal is also known at this point as we know the terminals to be either an input, output or a bidirectional pin. For a unidirectional current flow between an input-output pin pair of a net, we follow the principle described in Section 4.4 before, while for a pair of bidirectional pins, the candidate route is chosen by modifying the cost function slightly. In that case, the second and third central moments are evaluated considering the current flow in either direction for each of the candidate routes. The route, which yields the minimum cost in both the cases is selected the winner after doing a cumulative measure of the two moments.

We have considered only 2-pin nets that can be connected using either a 1-bend or a 2-bend route. Majority of the nets in a MCM circuit have only two pins. This can be observed from the 
data given in [10], of two industrial multi-chip module benchmarks. We see that for mcc1, $75.8 \%$ of the total number of nets are 2pin nets, while for mcc2 it is about $94.11 \%$. We focus on routing these two pin nets using our methodology. Multi-terminal nets can be broken down into a number of two-terminal nets as described by Borah et. al in [17], in their edge-based steiner-tree routing methodology. The resulting two terminal nets can then be routed using our methodology. The inspiration of using L-shaped and Zshaped technique find its origin in other related work by Kastner et.al in [1], [3] and by Smey et. al in [2]. A pre-specified step-size is used to generate ' $\mathrm{k}$ ' possible 2-bend candidate solutions for the current net that needs to be routed. Our implementation is flexible to accommodate other methods to generate candidate solutions as well. For 1-bend candidates, there can be at most two routes (either a upper-L or a Lower-L route) to choose from. As we inspect ' $\mathrm{k}+2$ ' possible candidates for each of the 'n' nets in our sequential algorithm, the routing complexity is $\Theta(k n)$, in contrast to $\Theta\left(k^{n}\right)$ complexity in the method described in [2]. We would also like to point out that the symbolic transfer function generation using SAPWIN is a 'one-time' process, occurs outside the algorithm loop and takes a few seconds for each of the templates.

\section{RESULTS AND DISCUSSIONS}

We have randomly distributed pairs of pins in our experiments and have routed the terminal pairs using our moment-driven routing algorithm. Our cost function is formulated based on the second and third order central moments $\left(\mu_{2}\right.$ and $\left.\mu_{3}\right)$. During moment evaluation we have accounted for the nearest neighbor capacitive and inductive coupling parasitics as well as the self resistance, capacitance and inductance of each net. We compare our routing methodology with the following competing approach.

Competing Approach: In this routing approach we have formulated a cost function, which takes into account the coupling capacitive parasitics between neighboring wires for each of the probable candidate routes during the route determination phase of a net. We decouple the competing approach from our moment-driven approach in the following ways.

1. The cost function does not include moment computations.

2. Resistive and Inductive (self and mutual) parasitics are ignored in the cost function.

3. Coupling capacitive parasitics are accounted in the cost function.

As the objective of the cost function of the competing approach is to minimize the coupling capacitive parasitics of the candidate route with its neighboring wires, it tries to find a route with maximal separation from its neighboring wires.

Figure 7(a), (b), (c) and (d) shows the step response for 200, 400, 700 and 900 nets using the two approaches. As the worst net determines the maximum speed of the circuit, we chose to do a comparative analysis on it. The worst net in our approach is the one with the highest cost based on the moment metrics, while the worst net in the competing approach is the one with the maximum capacitive coupling parasitics.

For the Hspice simulation, we extract all the parasitics (R, L, $\mathrm{C}, \mathrm{Cc}$ and $\mathrm{K}$ ) for the worst nets in both the approaches, even considering the mutual interactions with their neighboring nets. The rise time of the step response is set at $34 \mathrm{ps}$, which means a significant frequency of $0.34 / 34 p s=10 G H z$ [18]. The significant frequency should not be confused with the operating frequency, as it is dependent only on the rise time and not on the clock period. In other words, it signifies the edge rate of transition which helps to capture the effect of reactive components ( $\mathrm{L}$ and $\mathrm{C}$ ) in the circuit. More in-depth analysis of this measure is given in [19].
The graphs clearly show that our method results in better performance for the worst net. While the competing method resulted in overdamped (more delay) waveforms for the 200, 400, 700 and 900 routing cases, we were able to obtain a better quality response for all the routing cases using our approach. The inferior response for the competing approach can also be analyzed intuitively. As minimization of coupling parasitics tries to maximize the separation between each candidate route and its neighbors, the technique might not yield good results as the routing density increases. For future nets, there might not be enough space left to maximize the separation objective and may result in undesirable increase in coupling parasitics and an overdamped (decayed) response. Due to the area constraint on the routing region, we observe that as the density of nets increases (from 200 to 900), the output response progressively deteriorates.

The proposed method obtains a routing solution based on a cost function that finds the best trade-off between ringing $\left(\mu_{3}\right)$ and delay $\left(\mu_{2}\right)$. This ensures a signal response with better signal quality in terms of rise time, settling time, signal overshoots and undershoots. For the responses in Figure 7, we observe that the signal settles above $1.7 \mathrm{~V}(90 \%$ of $1.3 \mathrm{~V})$ after at most one undershoot. In the 400 and 900 routing instances it settles even faster, since the undershoot level does not go below the $1.7 \mathrm{~V}$ threshold mark. As the competing method is not driven by this cost function, the routing solution is not guaranteed to be of good quality and can suffer from either a signal underdamp or an overdamp. Similar promising results are also obtained for the $300,500,600,800$ and 1000 net cases.

In order to make the benchmarks more stringent, we kept the area of the routing region for all the benchmarks constant. In reality, for MCM circuits as the number of nets increases, the chip area and the area of the routing region increases too [10],[20]. 'mcc1' with 608 2-pin nets and 'mcc2' with 6699 2-pin nets are distributed on a $45 \times 45$ and a $152.4 \times 152.4 \mathrm{~mm}^{2}$ substrate respectively. In order to make the routing problem more intensive and stringent, we kept the routing area constant across our benchmarks.

The horizontal and vertical segments of the routes are in different metal layers and the minimum width and spacing rules required to avoid DRC violations are implemented in the routing methodology. The routing algorithm has been developed in $\mathrm{C}++$, and we have used SAPWIN to generate the one-time symbolic transfer functions for the templates used. The real time taken to complete the routing of all the nets in each instance is comparable for both our momentdriven and the competing approach. For the 1000 net routing case it took 21.5 minutes using our approach while it took 18.7 minutes using the competing approach on a Sun Ultra system with $256 \mathrm{MB}$ memory. The difference in time is due to the extra computation time required to calculate the moments in our approach. Thus without a significant increase in time, we obtain a solution with better signal quality and response. Direct comparison of our results with [10] is not possible as [10] dealt with only a few percentage of nets with steiner tree connections and did not report on the worst case considering the routing of all the nets.

\subsection{Discussion on Worst Case Analysis}

We have assumed the signals in all the wires switching at the same time to model a worst case scenario. Recent trends highlight the importance of stochastic modeling of the switching in wires, arguing that in reality not all wires switch simultaneously and considering simultaneous switching might lead to the underestimation of the rise time of the signals. Though much attention is being given to the stochastic switching distribution, we considered the worst case scenario with the reasoning that the routing obtained under this condition will also be effective under stochastic switching. 

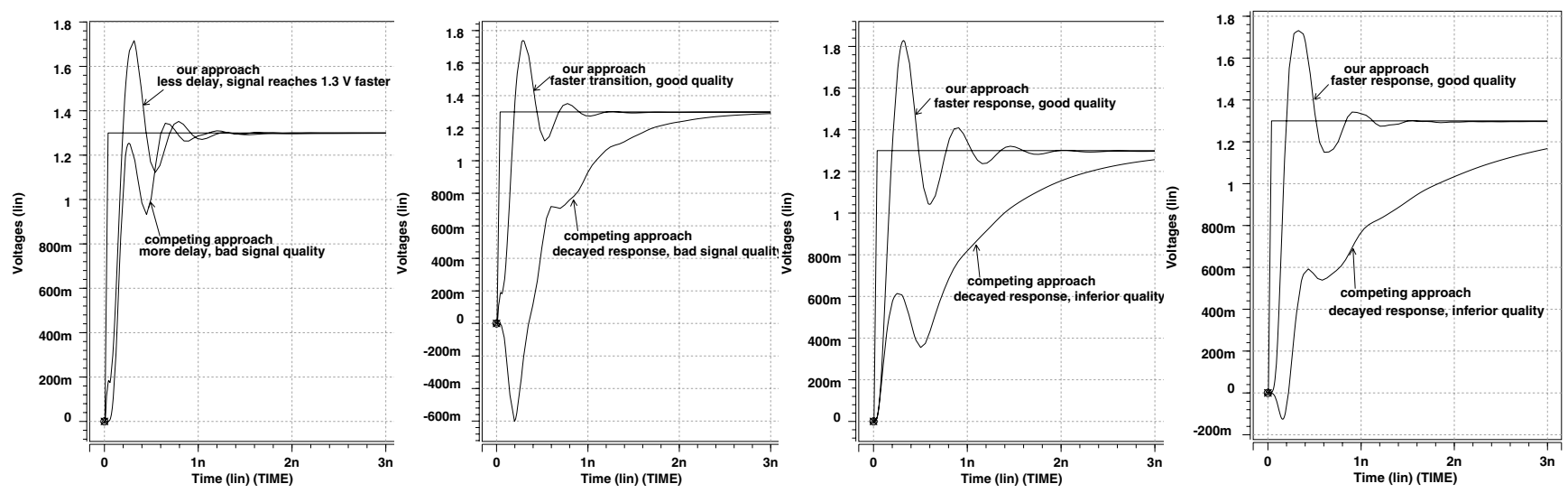

Figure 7: Hspice simulation result for the worst net in our and competing approach for (a) 200 (b) 400 (c) 700 and (d) 900 nets

\section{CONCLUSION}

Performance-driven routing is increasingly becoming important as circuit and multi-chip-module (MCM) designs progress towards the deep-submicron (DSM) regime. Interconnect parasitic optimization cannot remain restricted to a simple RC or a RLC model. At multi-GHz frequencies, the crosstalk among interacting wires become important. In this paper, we have proposed a routing methodology, which takes into account the inductive and capacitive coupling parasitics between neighboring wires during the cost function evaluation of the moment-driven routing process. The proposed approach ensures a routing solution for a number of randomly distributed pin-pairs, with good signal quality and minimum rise time delay. The trade-off between ringing and delay is effectively captured using a moment-driven cost function. Routing obtained using only capacitive coupling in the cost function precludes the trade-off and yields an inferior solution in terms of signal quality and delay. Acknowledgement: We thank National Science Foundation for partly supporting this work under the award number CCF-0429717.

\section{REFERENCES}

[1] R. Kastner, E. Bozorgzadeh and M. Sarrafzadeh. Coupling Aware Routing. In International ASIC/SOC Conference, pages 392 - 396, 2000.

[2] R. M. Smey, B. Swartz and P H. Madden. Crosstalk Reduction in Area Routing. In Design Automation and Test in Europe Conference, pages 862 - 867, 2003.

[3] R. Kastner, E. Bozorgzadeh and M. Sarrafzadeh. Predictable Routing. In International Conference on Computer Aided Design, pages $110-113,2000$.

[4] R. T. Hadsell and P. M. Madden. Improved Global Routing through Congestion Estimation. In Design Automation Conference, pages $28-31,2003$.

[5] J. Cong and P. Madden. Performance Driven Multi-Layer General Area Routing for PCB/MCM Designs. In Design Automation Conference, pages 356 - 361, 1998.

[6] T. Ho et. al. A Fast Crosstalk- and Performance-Driven Multilevel Routing System. In International Conference on Computer Aided Design, pages 382 - 387, 2003.

[7] J. Lillis et.al. New Performance Driven Routing Techniques With Explicit Area/Delay Tradeoff and Simultaneous Wire Sizing. In Design Automation Conference, pages 395 - 400,
1996.

[8] S. Hur, A. Jagannathan and J. Lillis. Timing-Driven Maze Routing. In Tran. on Computer-Aided Design of Integrated Circuits and Systems, pages 234 - 241, 2000.

[9] J. Hu and S. Sapatnekar. A Timing-Constrained Simultaneous Global Routing Algorithm. In Tran. on Computer-Aided Design of Integrated Circuits and Systems, pages 1025 - 1036, 2002.

[10] J. Cong, C. Koh and P. Maddden. Interconnect Layout Optimization Under Higher Order RLC Model for MCM Designs. In Tran. on Computer-Aided Design of Integrated Circuits and Systems, pages 1455 - 1463, 2001.

[11] H. B. Bakoglu. Circuits, Interconnections, and Packaging for VLSI. Addison-Wesley, 1990.

[12] A. Bhaduri and R. Vemuri. Moment-Driven Coupling-Aware Routing Methodology. In Great Lakes Symposium on VLSI, pages $390-395,2005$.

[13] R. Gupta, B. Krauter, L. T. Pileggi. Transmission Line Synthesis via Constrained Multivariable Optimization. In Tran. on Computer-Aided Design of Integrated Circuits and Systems, pages 6-19, 1997.

[14] Y. Lu et. al. Min/Max On-Chip Inductance Models and Delay Metrics. In Design Automation Conference, pages 341-346, 2001.

[15] M. Xu, L. He. An Efficient Model for Frequency-Dependent On-Chip Inductance. In Great Lakes Symposium on VLSI, 2001.

[16] A. Bhaduri and R. Vemuri. Inductive and Capacitive Coupling Aware Routing Methodology Driven by a Higher Order RLCK Moment Metric. In Design Automation and Test in Europe, pages 922 - 923, 2005.

[17] M. Borah et. al. An edge-based heuristic for Steiner routing. In Tran. on Computer-Aided Design of Integrated Circuits and Systems, pages 1563 - 1568, 1994.

[18] C. K. Cheng, J. Lillis, S. Lin and N. Chang. Interconnect Analysis and Synthesis. John Wiley \& Sons, Inc, 2000.

[19] H. W. Johnson and M. Graham. High-Speed Digital Design A Handbook of Black Magic. Prentice Hall, 1993.

[20] K. Khoo and J. Cong. An Efficient Multilayer MCM Router Based on Four-Via Routing. In Tran. on Computer-Aided Design, pages 1277 - 1290, 1995. 